\title{
Dihydroartemisinin induces endothelial cell anoikis through the activation of the JNK signaling pathway
}

\author{
JIAO ZHANG ${ }^{1}$, LING GUO $^{2}$, XIA ZHOU ${ }^{3}$, FENGYUN DONG $^{2}$, LIQUN LI $^{2}$, \\ ZUOWANG CHENG ${ }^{4}$, YINGHUA XU ${ }^{4}$, JIYONG LIANG ${ }^{5}$, QI XIE ${ }^{2}$ and $\mathrm{JU}_{\text {LIU }}^{2}$
}

${ }^{1}$ Department of Infectious Diseases, Shandong Provincial Hospital Affiliated to Shandong University, Jinan, Shandong 250021;

${ }^{2}$ Medical Research Center; ${ }^{3}$ Department of Traditional Chinese Medicine, Shandong Provincial Qianfoshan Hospital,

Shandong University, Jinan, Shandong 250014; ${ }^{4}$ Taishan Medical College, Taian, Shandong 271021, P.R. China;

${ }^{5}$ Department of Systems Biology, The University of Texas MD Anderson Cancer Center, Houston, TX 77030, USA

Received February 4, 2015; Accepted July 7, 2016

DOI: $10.3892 / \mathrm{ol} .2016 .4870$

\begin{abstract}
Angiogenesis is required for the growth and metastasis of solid tumors. The anti-malarial agent dihydroartemisinin (DHA) demonstrates potent anti-angiogenic activity, but the underlying molecular mechanisms are not yet fully understood. During the process of angiogenesis, endothelial cells migrating from existing capillaries may undergo programmed cell death after detaching from the extracellular matrix, a process that is defined as anchorage-dependent apoptosis or anoikis. In the present study, DHA-induced cell death was compared in human umbilical vein endothelial cells (HUVECs) cultured in suspension and attached to culture plates. In suspended HUVECs, the cell viability was decreased and apoptosis was increased with the treatment of $50 \mu \mathrm{M}$ DHA for $5 \mathrm{~h}$, while the same treatment did not affect the attached HUVECs. In addition, $50 \mu \mathrm{M}$ DHA increased the phosphorylation of c-Jun N-terminal kinase (JNK) in suspended HUVECs, but not in attached HUVECs, for up to $5 \mathrm{~h}$ of treatment. The JNK inhibitor, SP600125, reversed DHA-induced cell death in suspended HUVECs, suggesting that the JNK pathway may mediate DHA-induced endothelial cell anoikis. The data from the present study indicates a novel mechanism for understanding the anti-angiogenic effects of DHA, which may be used as a component for chemotherapy.
\end{abstract}

\section{Introduction}

Angiogenesis is an important physiological process, through which novel blood vessels develop from existing vessels (1).

Correspondence to: Professor Ju Liu or Dr Qi Xie, Medical Research Center, Shandong Provincial Qianfoshan Hospital, Shandong University, 16766 Jingshi Road, Jinan, Shandong 250014, P.R. China

E-mail: ju.liu@sdu.edu.cn

E-mail: xieqi2012@163.com

Key words: dihydroartemisinin, c-Jun N-terminal kinase signaling, angiogenesis, endothelial cell, anoikis
Persistent and uncontrolled angiogenesis is involved in the pathogenesis of rheumatoid arthritis, atherosclerosis, diabetes, ocular retinopathy and tumors $(2,3)$. In particular, tumor angiogenesis is crucial for solid tumor growth, invasion and metastasis (4). During tumor growth, the production of angiogenic factors, including members of the vascular endothelial growth factor (VEGF) and fibroblast growth factor (FGF) families, from tumor cells results in the induction of capillary spouting and the subsequent growth of novel vessels in tumors from surrounding host vessels $(3,5)$. As the vessels in the tumor are tortuous, with sluggish flow and a lack of surrounding pericytes, tumor cells readily invade into novel vessels and form tumor emboli (3). In addition, tumor cells seed in distance organs, where they undergo secondary angiogenesis. Therefore, the disruption of angiogenesis has been considered as an effective therapeutic strategy in the treatment of solid tumors (6).

Angiogenesis is a multi-step process involving endothelial cell activation, proliferation, migration, differentiation, maturation and tube formation (3,7). Endothelial cells are central for angiogenesis. Previous studies have demonstrated that the inhibition of endothelial cell death is an essential prerequisite to maintain vascular remodeling and angiogenesis (8). There are several types of morphological distinct cell death exhibited by endothelial cells (9). Adhesion to the extracellular matrix (ECM) is a crucial for survival of endothelial cells (7). During tumor angiogenesis, the vascular basement membrane is degraded after endothelial cell activation, and then the endothelial cells migrate into the subendothelial space without attachment to the ECM (8). Similarly to other anchorage-dependent cells, endothelial cells undergo cell death after detachment from the underlying ECM, which is defined as anoikis (10).

Anti-malarial agent dihydroartemisinin (DHA) is a semi-synthetic derivative of artemisinin that is extracted from the herbaceous plant, Artemisia annua (11). DHA exhibits potent antitumor and anti-angiogenesis effects and has therefore emerged as a potential component for cancer chemotherapies (12). DHA inhibits endothelial cell proliferation and migration via the downregulation of the nuclear factor- $\mathrm{\kappa} B$ and extracellular signal-regulated kinase (ERK) signaling pathways (13-15). Several studies have suggested that the anti-angiogenic effects of DHA may be partly associated with 
its role in promoting the apoptosis of endothelial cells $(12,16)$. However, the effects of DHA on endothelial cell anoikis have not yet been studied.

In the present study, human umbilical vein endothelial cells (HUVECs) in suspension were used as a model for anoikis. The cells in suspension or attached to culture plates were treated with DHA. The cell death of HUVECs in these two models was determined. Notably, $5 \mathrm{~h}$ treatment of $50 \mu \mathrm{M}$ DHA significantly increased the cell death of HUVECs in suspension, but not for HUVECs attached to the plates. In addition, DHA specifically activated the c-Jun N-terminal kinase (JNK) pathway in suspended HUVECs, and the inhibition of the JNK pathway reversed the cell death of HUVECs in suspension. These results suggest that DHA promotes endothelial cell anoikis via the activation of the JNK pathway.

\section{Materials and methods}

Cell culture. HUVECs were purchased from Lonza Group Ltd. (Basel, Switzerland) and were cultured in endothelial basal cell medium-2 supplemented with EGM-2-MV bullet kit (Lonza Group Ltd.) and antibiotics (100 international units/ml penicillin and $100 \mu \mathrm{g} / \mathrm{ml}$ streptomycin). The cells were cultured at $37^{\circ} \mathrm{C}$ in a humidified atmosphere containing $5 \% \mathrm{CO}_{2}$. The HUVECs grown on culture plates were used as attached cells. A group of confluent cells were trypsinized for $2 \mathrm{~min}$ and detached to form single cell suspension. These cells were cultured in suspension by slow rotation in culture flasks and collected at $5 \mathrm{~h}$ as suspended HUVECs. DHA (Sigma-Aldrich, St. Louis, MO, USA) and the JNK inhibitor, SP600125 (Cell Signaling Technology, Inc., Danvers, MA, USA), were dissolved in dimethyl sulfoxide (DMSO).

Trypan blue exclusion assay. Cell viability was assessed at $5 \mathrm{~h}$ after $50-\mu \mathrm{M}$ DHA treatment. The single cell suspensions were prepared and diluted 1:1 with $0.4 \%$ trypan blue $(\mathrm{w} / \mathrm{v}$ in 0.9\% NaCl; Santa Cruz Biotechnology, Dallas, TX, USA). The dye-free cells were calculated under a light microscope.

Flow cytometry. The cell death of HUVECs induced by DHA was detected by using Annexin V-fluorescein isothiocyanate (FITC) and propidium iodide (PI) staining (NeoBioscience, Shenzhen, China), according to the manufacturer's instructions. Briefly, single cell suspensions from attached or suspended HUVECs were prepared, washed with phosphate-buffered saline (PBS) and resuspended in binding buffer containing Annexin V-FITC $(0.25 \%)$ and PI $(1 \mu \mathrm{g} / \mathrm{ml})$. An aliquot of $1 \times 10^{5}$ cells were examined using a FACSAria II flow cytometer (BD Biosciences, San Jose, CA, USA). The percentages of positive cells were analyzed using the FACSDiva version 6.0 acquisition and analysis software (BD Biosciences).

Western blotting. HUVECs were collected and washed with cold PBS, then lysed in radioimmunoprecipitation assay buffer [20 mM Tris (pH 7.5), $150 \mathrm{mM} \mathrm{NaCl}, 50 \mathrm{mM} \mathrm{NaF}, 1 \%$ NP40, $0.1 \%$ deoxycholate, $0.1 \%$ sodium dodecyl sulfate (SDS), $1 \mathrm{mM}$ ethylenediaminetetraacetic acid, $1 \mathrm{mM}$ phenylmethane sulfonyl fluoride and $1 \mathrm{mg} / \mathrm{ml}$ leupeptin]. Protein concentrations were determined using bicinchoninic acid assay (Bio-Rad Laboratories, Inc., Hercules, CA, USA). Equal amounts of protein were separated by $8 \%$ SDS-polyacrylamide gel electrophoresis and transferred to the polyvinylidene fluoride (PVDF) membrane. After being blocked with 5\% skimmed milk overnight, the PVDF membranes were incubated with primary antibodies in PBS-Tween at $4^{\circ} \mathrm{C}$. The primary antibodies used were rabbit monoclonal anti-phosphorylated- (p-)JNK (1:500; 4668; Cell Signaling Technology, Inc.), rabbit polyclonal anti-JNK (1:500; 9252; Cell Signaling Technology, Inc.) and rabbit polyclonal anti-glyceraldehyde 3-phosphate dehydrogenase (GAPDH; 1:5,000; SAB2100894, Sigma-Aldrich). Immunoreactivity was visualized by using a horseradish peroxidase-conjugated goat anti-rabbit immunoglobulin G secondary antibody $(1: 2,000$; 7074; Cell Signaling Technology, Inc.) and a chemiluminescence kit (Pierce ECL; Thermo Fisher Scientific, Inc., Waltham, MA USA). The densitometry analyses were performed using ImageJ software (National Institutes of Health, Bethesda, MD, USA). GAPDH levels were used as controls for protein loading.

Statistical analyses. Each experiment was performed at least 3 times. Statistical analyses were performed using SPSS version 11.5 (SPSS, Inc., Chicago, IL, USA). The results are presented as the mean \pm standard deviation. A Student's $t$-test was used for statistical comparisons between two groups. $\mathrm{P}<0.05$ was considered to indicate a statistically significant difference.

\section{Results}

DHA inhibits the cell viability of suspended endothelial cells. The effects of DHA on the cell viability of HUVECs were evaluated by trypan blue exclusion assay. After $5 \mathrm{~h}$ in suspension, the cell viability of HUVECs in the non-treatment group was significantly decreased compared with the attached HUVECs $(88.3 \pm 4.6 \%$ vs. $73.6 \pm 3.1 \%$; $\mathrm{P}=0.03)$. The percentage of viable cells in attached HUVECs was not affected by $50 \mu \mathrm{M}$ DHA after $5 \mathrm{~h}$ incubation $(88.3 \pm 4.6 \%$ vs. $90.7 \pm 6.1 \%$; $\mathrm{P}=0.23)$ (Fig. 1A). However, the percentage of viable cells in suspended HUVECs was significantly decreased after the same DHA treatment (73.6 $\pm 3.1 \%$ vs. $58.7 \pm 8.1 \%$; $\mathrm{P}=0.02$ ) (Fig. $1 \mathrm{~B}$ ). Therefore, $\mathrm{DHA}$ is likely to inhibit the cell viability of suspended endothelial cells but not attached endothelial cells.

DHA induces apoptosis in suspended endothelial cells. To investigate the apoptotic status of the unviable cells, HUVECs with DHA treatment were analyzed by flow cytometry with Annexin V and PI staining. Consistent with viability assays, increased apoptosis was observed in suspended HUVECs compared with attached HUVECs in the non-treatment group ( $15.9 \pm 2.1 \%$ vs. $27.1 \pm 3.5 \%$; $\mathrm{P}=0.04)$, suggesting that the suspended endothelial cells undergo anoikis. DHA did not alter apoptosis in attached HUVECs $(15.9 \pm 2.1 \%$ vs. $16.3 \pm 1.7 \%$; $\mathrm{P}=0.31$ ) (Fig. 1C), but induced a significant increase of the apoptosis in suspended HUVECs $(27.1 \pm 3.5 \%$ vs. $39.3 \pm 4.4 \% ; \mathrm{P}=0.02)$ (Fig. 1D). This indicates that DHA specifically enhances anoikis in endothelial cells.

DHA activates the JNK pathway in suspended endothelial cells. JNK is one of the major components of the mitogen-activated protein kinase cascade, and participates in cell death signaling pathways (17). Studies have suggested that the JNK pathway may mediate anoikis in epithelial cells $(18,19)$. Therefore, the 
A

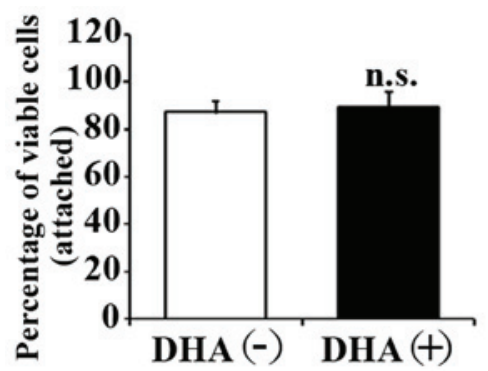

C

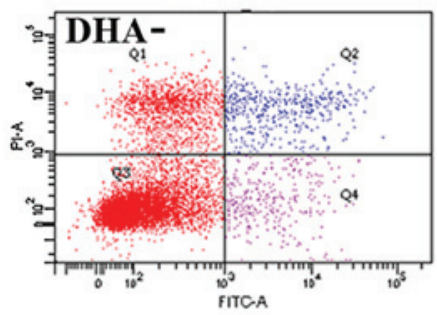

D

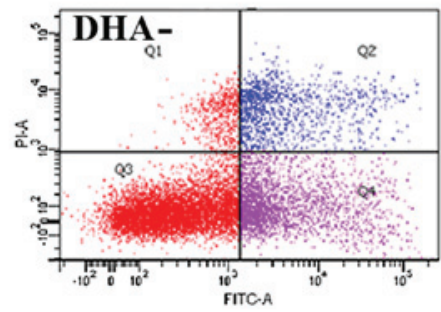

B
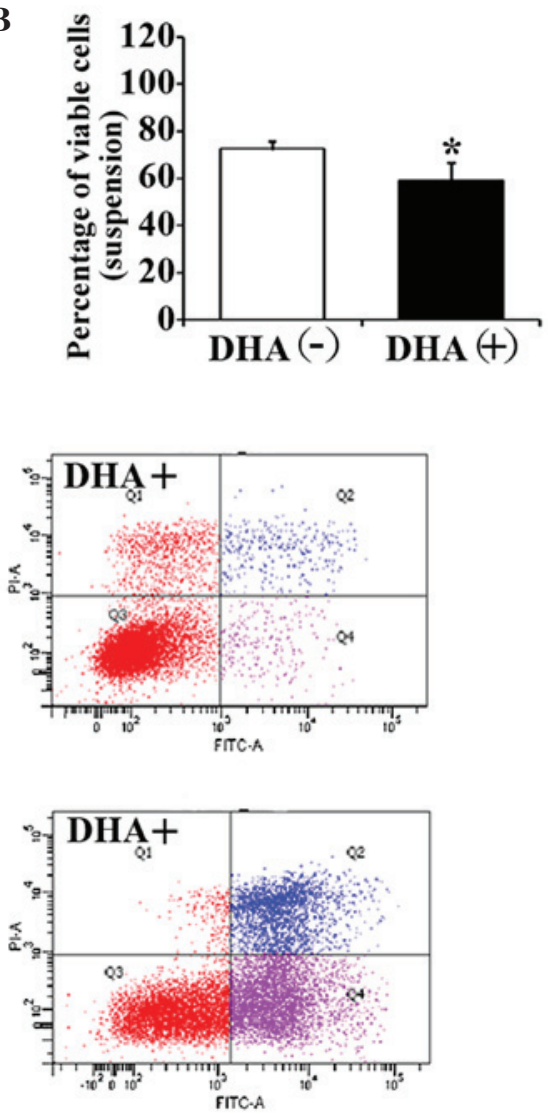

Figure 1. Viability and apoptosis of HUVECs treated with DHA. Percentages of viable cells from (A) attached or (B) suspended HUVECs treated with $50 \mu \mathrm{M}$ DHA for $5 \mathrm{~h} ; \mathrm{n}=4$. "P<0.05 vs. DHA (-). Representative images of (C) attached or (D) suspended HUVECs treated with $50 \mu \mathrm{M}$ DHA for $5 \mathrm{~h}$ by flow cytometry analysis with Annexin V/PI-staining. DHA, dihydroartemisinin; HUVEC, human umbilical vein endothelial cell; n.s., non-significant; PI, propidium iodide; FITC, fluorescein isothiocyanate.

present study examined the activation of JNK in DHA treated HUVECs by western blotting. As shown in Fig. 2A and B, for up to $5 \mathrm{~h}$ incubation with $50 \mu \mathrm{M}$ DHA, the level of p-JNK remained unchanged in attached HUVECs $(\mathrm{P}=0.13)$. However, the same treatment of DHA significantly increased $\mathrm{p}-\mathrm{JNK}$ in suspended HUVECs ( $\mathrm{P}=0.01)$ (Fig. $2 \mathrm{C}$ and $\mathrm{D}$ ), suggesting that DHA activates JNK pathway in suspended endothelial cells but not in attached endothelial cells.

JNK inhibitor SP600125 reverses HUVEC anoikis induced by DHA. SP600125 is a cell-permeable and selective inhibitor of the JNK pathway (20). To further verify the role of the JNK signaling pathway in the cell death of suspended HUVECs, $10 \mu \mathrm{M}$ SP600125 was applied to suspended HUVECs for $1 \mathrm{~h}$ prior to DHA treatment. Fig. 3A indicates that SP600125 successfully prevented the increase of p-JNK. SP600125 treatment abrogated the decrease of viable cells in suspended HUVECs treated with DHA ( $74.6 \pm 4.5 \%$ vs. $78.9 \pm 6.6 \% ; \mathrm{P}=0.19)$ (Fig. 3B). SP600125 also attenuated DHA-induced apoptosis in suspended HUVECs $(28.6 \pm 2.5 \%$ vs. $30.3 \pm 4.9 \%$; $\mathrm{P}=0.12)$ (Fig. 3C). These results suggest that JNK signaling pathway mediates DHA induced anoikis in endothelial cells.

\section{Discussion}

DHA possesses strong anti-angiogenic activities, but the molecular mechanisms are not yet fully understood $(13,14,21)$.
The present study examined the effects of DHA on endothelial cell death in attached and suspended HUVECs. After $5 \mathrm{~h}$ incubation, $50 \mu \mathrm{M}$ DHA induced cell death in suspended endothelial cells, but not in attached endothelial cells. In addition, DHA increased the expression of p-JNK, and blocking the JNK pathway abrogated DHA-induced cell death in suspended endothelial cells. The present study indicates that DHA induces endothelial cell anoikis via activation of the JNK pathway.

Anoikis is induced by lack of correct cell or ECM attachment, and anoikis resistance is important for tumor metastasis (10). During tumor angiogenesis, a group of endothelial cells migrate across the basement membrane, leading to insufficient cell-matrix interactions and subsequent anoikis (1). Consistent with the findings of other reports, the present study revealed the additional occurrence of cell death in untreated endothelial cells in suspension, a model in which endothelial cells are completely detached from the ECM. Endothelial cell anoikis is crucial for tumor angiogenesis and presents a target for anti-angiogenic therapy. In the current study, treatment with a low concentration of DHA $(50 \mu \mathrm{M})$ for a short-term exposure time $(5 \mathrm{~h})$ was observed to induce cell death in suspended HUVECs. The same treatment did not affect cell survival of attached HUVECs. This suggests that DHA inhibits angiogenesis, which is at least partially due to the induction of endothelial cell anoikis.

The JNK pathway, which appears to be activated by detachment from the ECM, is critical for tumor cell apoptosis (22). However, its role in anoikis has been controversial. Several studies 
A

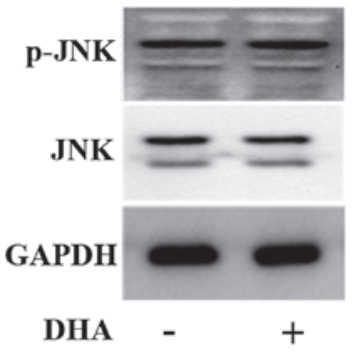

C

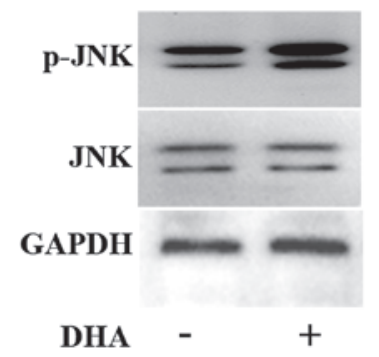

B

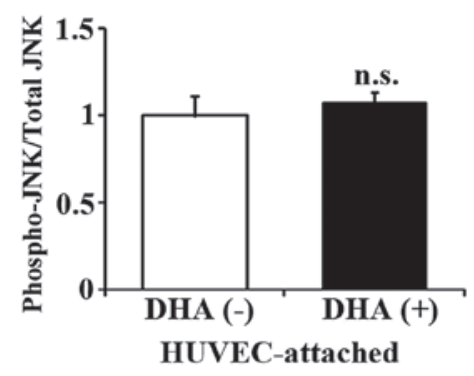

D

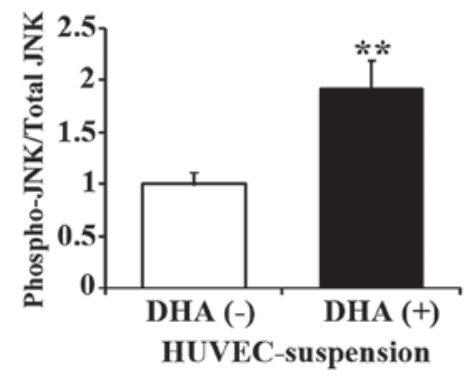

Figure 2. DHA activates JNK signaling pathway in suspended HUVECs. (A) Representative immunoblots and (B) densitometry analysis of p-JNK and JNK in attached HUVECs treated with $50 \mu \mathrm{M}$ DHA for $5 \mathrm{~h} ; \mathrm{n}=3$. (C) Representative immunoblots and (D) densitometry analysis of p-JNK and JNK in suspended HUVECs treated with $50 \mu \mathrm{M}$ DHA for $5 \mathrm{~h} ; \mathrm{n}=3$. ** $\mathrm{P}<0.01$ vs. DHA (-). DHA, dihydroartemisinin; JNK, c-Jun N-terminal kinase; p-JNK, phosphorylated-JNK; GAPDH, glyceraldehyde 3-phosphate dehydrogenase; HUVEC, human umbilical vein endothelial cell; n.s., non-significant.

A

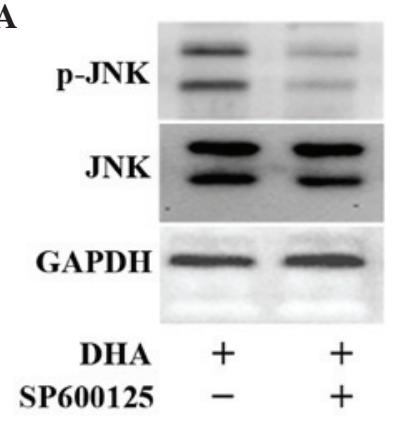

C

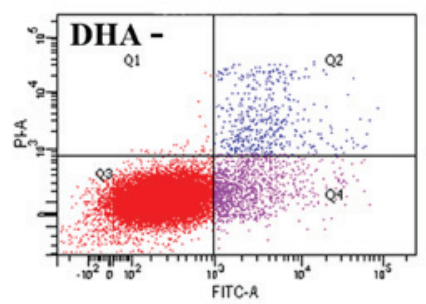

B
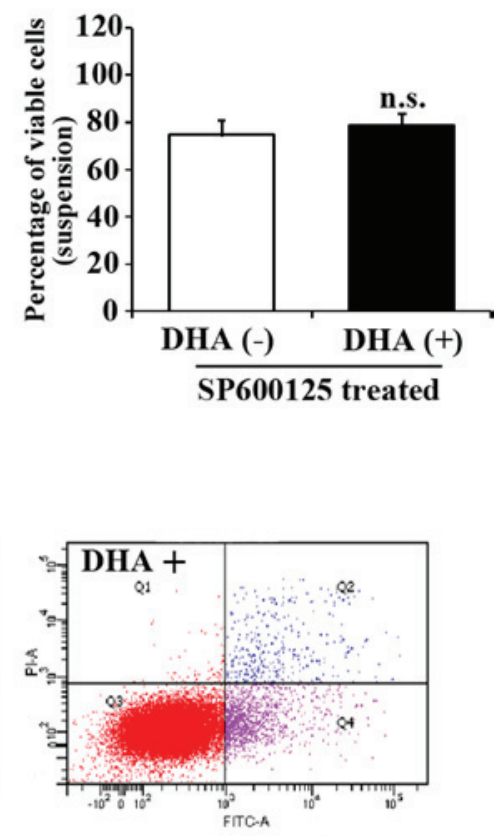

SP600125 treated HUVECs in suspension

Figure 3. JNK inhibitor, SP600125, reverses the viability and apoptosis of suspended HUVECs induced by DHA. (A) Representative immunoblots of p-JNK and JNK in suspended HUVECs treated with DHA and SP600125. (B) Percentage of viable cells from suspended HUVECs treated with DHA and SP600125; $\mathrm{n}=4$. (C) Representative images of flow cytometry analyses of Annexin V/PI-staining in suspended HUVECs treated with DHA and SP600125. DHA, dihydroartemisinin; JNK, c-Jun N-terminal kinase; p-JNK, phosphorylated-JNK; GAPDH, glyceraldehyde 3-phosphate dehydrogenase; HUVEC, human umbilical vein endothelial cell; n.s., non-significant; PI, propidium iodide; FITC, fluorescein isothiocyanate.

have reported that the activation of the JNK signaling pathway mediates anoikis in cancer cell lines $(19,23,24)$. In addition, B cell lymphoma-2 has been found to suppress the suspension-induced activation of JNK signaling, which requires the proteolytic function associated with interleukin-1- $\beta$-converting enzyme (18). Conversely, the study by Khwaja et al (25) reported that the
JNK pathway is not associated with anoikis in epithelial cells. In the present study, DHA was demonstrated to promote anoikis in suspended HUVECs through activation of the JNK signaling pathway. In endothelial cells, detachment results in a rapid rise in the level of reactive oxygen species (ROS), which modulate the activity of the JNK signaling pathway (26). DHA increases 
the level of ROS in several cancerous cell lines $(27,28)$. Another artemisinin derivative, artesunate, significantly inhibits corneal neovascularization by inducing ROS-dependent apoptosis in vascular endothelial cells (29). Therefore, DHA may induce an increase in ROS and then activate then JNK signaling pathway in suspended HUVECs.

The JNK pathway may also interact with focal adhesion kinase (FAK) signaling in mediating anoikis. FAK is a key component of cell-substratum adhesions, and disruption of FAK signaling results in a loss of substrate adhesion and anoikis in endothelial cells (30). Glucocorticoids induce osteocyte anoikis by blocking FAK signaling and activating JNK. In addition, FAK blocks the ras-related $\mathrm{C} 3$ botulinum toxin substrate $1 / \mathrm{JNK}$ pathway in vascular smooth muscle cells (31). However, in lung adenocarcinoma cells, FAK regulates anoikis independently of the JNK pathway (32). DHA directly decreases the level of p-FAK in ovarian cancer cells (33). Thus, the role of FAK signaling in DHA induced anoikis requires further investigation.

In summary, the present study demonstrates that DHA induces endothelial cell anoikis, which is mediated by the activation of the JNK pathway. DHA may be considered as a promising angiogenesis inhibitor for clinical application. The findings of the present study will aid the current understanding of the molecular mechanisms underlying the anti-angiogenic effects of DHA.

\section{Acknowledgements}

The present study was supported by the Medical Science and Technology Development Plan of Shandong Province (Jinan, China; grant no. 2013WS0137). The authors are grateful for the support provided to Professor Ju Liu from the Shandong Taishan Scholarship (Jinan, China).

\section{References}

1. Kerbel RS: Tumor angiogenesis. N Engl J Med 358: 2039-2049, 2008.

2. Folkman J: Angiogenesis in cancer, vascular, rheumatoid and other disease. Nat Med 1: 27-31, 1995.

3. Carmeliet P: Mechanisms of angiogenesis and arteriogenesis. Nat Med 6: 389-395, 2000

4. Folkman J: Tumor angiogenesis: Therapeutic implications. N Engl J Med 285: 1182-1186, 1971.

5. Liu J, Deutsch U, Jeong J and Lobe CG: Constitutive notch signaling in adult transgenic mice inhibits bFGF-induced angiogenesis and blocks ovarian follicle development. Genesis 52: 809-816, 2014.

6. Kim KJ, Li B, Winer J, Armanini M, Gillett N, Phillips HS and Ferrara N: Inhibition of vascular endothelial growth factor-induced angiogenesis suppresses tumour growth in vivo. Nature 362 841-844, 1993.

7. Hanahan D: Signaling vascular morphogenesis and maintenance. Science 277: 48-50, 1997.

8. Aoudjit F and Vuori K: Matrix attachment regulates Fas-induced apoptosis in endothelial cells: A role for c-flip and implications for anoikis. J Cell Biol 152: 633-643, 2001.

9. Green DR and Llambi F: Cell death signaling. Cold Spring Harb Perspect Biol 7: pii: a006080, 2015.

10. Gilmore AP: Anoikis. Cell Death Differ 12 (Suppl 2): 1473-1477, 2005.

11. Lee J, Zhou HJ and Wu XH: Dihydroartemisinin downregulates vascular endothelial growth factor expression and induces apoptosis in chronic myeloid leukemia K562 cells. Cancer Chemother Pharmacol 57: 213-220, 2006.
12. Ho WE, Peh HY, Chan TK and Wong WS: Artemisinins: Pharmacological actions beyond anti-malarial. Pharmacol Ther 142: 126-139, 2014.

13. Dong F, Zhou X, Li C, Yan S, Deng X, Cao Z, Li L, Tang B, Allen TD and Liu J: Dihydroartemisinin targets VEGFR2 via the NF- $\kappa$ B pathway in endothelial cells to inhibit angiogenesis. Cancer Biol Ther 15: 1479-1488, 2014.

14. Dong F, Tian H, Yan S, Li L, Dong X, Wang F, Li J, Li C, Cao Z, Liu X and Liu J: Dihydroartemisinin inhibits endothelial cell proliferation through the suppression of the ERK signaling pathway. Int J Mol Med 35: 1381-1387, 2015

15. Guo L, Dong F, Hou Y, Cai W, Zhou X, Huang AL, Yang M, Allen TD and Liu J: Dihydroartemisinin inhibits vascular endothelial growth factor-induced endothelial cell migration by a p38 mitogen-activated protein kinase-independent pathway. Exp Ther Med 8: 1707-1712, 2014.

16. Chen HH, Zhou HJ, Wang WQ and Wu GD: Antimalarial dihydroartemisinin also inhibits angiogenesis. Cancer Chemother Pharmacol 53: 423-432, 2004

17. Liu J and Kapron CM: Differential induction of MAP kinase signalling pathways by cadmium in primary cultures of mouse embryo limb bud cells. Reprod Toxicol 29: 286-291, 2010.

18. Frisch SM, Vuori K, Kelaita D and Sicks S: A role for Jun-N-terminal kinase in anoikis; suppression by bcl-2 and crmA. J Cell Biol 135: 1377-1382, 1996.

19. Cardone MH, Salvesen GS, Widmann C, Johnson G and Frisch SM: The regulation of anoikis: MEKK-1 activation requires cleavage by caspases. Cell 90: 315-323, 1997.

20. Bennett BL, Sasaki DT, Murray BW, O'Leary EC, Sakata ST, Xu W, Leisten JC, Motiwala A, Pierce S, Satoh Y, et al: SP600125, an anthrapyrazolone inhibitor of Jun N-terminal kinase. Proc Natl Acad Sci USA 98: 13681-13686, 2001.

21. Oh S, Jeong IH, Shin WS and Lee S: Growth inhibition activity of thioacetal artemisinin derivatives against human umbilical vein endothelial cells. Bioorg Med Chem Lett 13: 3665-3668, 2003.

22. Leppa S and Bohmann D: Diverse functions of JNK signaling and c-Jun in stress response and apoptosis. Oncogene 18: 6158-6162, 1999.

23. Zhang Y, Rivera Rosado LA, Moon SY and Zhang B: Silencing of D4-GDI inhibits growth and invasive behavior in MDA-MB-231 cells by activation of Rac-dependent p38 and JNK signaling. J Biol Chem 284: 12956-12965, 2009.

24. Krestow JK, Rak J, Filmus J and Kerbel RS: Functional dissociation of anoikis-like cell death and activity of stress activated protein kinase. Biochem Biophys Res Commun 260: 48-53, 1999.

25. Khwaja A and Downward J: Lack of correlation between activation of Jun-NH2-terminal kinase and induction of apoptosis after detachment of epithelial cells. J Cell Biol 139: 1017-1023, 1997.

26. Li AE, Ito H, Rovira II, Kim KS, Takeda K, Yu ZY, Ferrans VJ and Finkel T: A role for reactive oxygen species in endothelial cell anoikis. Circ Res 85: 304-310, 1999.

27. Kong R, Jia G, Cheng ZX, Wang YW, Mu M, Wang SJ, Pan SH, Gao Y, Jiang HC, Dong DL and Sun B: Dihydroartemisinin enhances Apo2L/TRAIL-mediated apoptosis in pancreatic cancer cells via ROS-mediated up-regulation of death receptor 5. PloS One 7: e37222, 2012.

28. Kim SJ, Kim MS, Lee JW, Lee CH, Yoo H, Shin SH, Park MJ and Lee SH: Dihydroartemisinin enhances radiosensitivity of human glioma cells in vitro. J Cancer Res Clin Oncol 132: 129-135, 2006.

29. Cheng R, Li C, Wei L, Li L, Zhang Y, Yao Y, Gu X, Cai W, Yang Z, Ma J, et al: The artemisinin derivative artesunate inhibits corneal neovascularization by inducing ROS-dependent apoptosis in vascular endothelial cells. Invest Ophthalmol Vis Sci 54: 3400-3409, 2013.

30. Lu Q and Rounds S: Focal adhesion kinase and endothelial cell apoptosis. Microvasc Res 83: 56-63, 2012.

31. Sundberg LJ, Galante LM, Bill HM, Mack CP and Taylor JM: An endogenous inhibitor of focal adhesion kinase blocks Rac1/JNK but not Ras/ERK-dependent signaling in vascular smooth muscle cells. J Biol Chem 278: 29783-29791, 2003.

32. Liu G, Meng X, Jin Y, Bai J, Zhao Y, Cui X, Chen F and Fu S: Inhibitory role of focal adhesion kinase on anoikis in the lung cancer cell A549. Cell Biol Int 32: 663-670, 2008.

33. Wu B, Hu K, Li S, Zhu J, Gu L, Shen H, Hambly BD, Bao S and Di W: Dihydroartiminisin inhibits the growth and metastasis of epithelial ovarian cancer. Oncol Rep 27: 101-108, 2012. 